\title{
Course Recommendation System
}

\author{
M. Rekha Sundari, PhD \\ Associate Professor \\ Dept of IT, ANITS \\ Visakhapatnam
}

\author{
G. Shreya \\ Student \\ Dept of IT, ANITS \\ Visakhapatnam
}

\author{
T. Jawahar \\ Student \\ Dept of IT, ANITS \\ Visakhapatnam
}

\begin{abstract}
Choice based credit system in higher education employs simple principle of students choosing courses of their interests. This learning platform makes students face difficulty in choosing electives, as the options available are multitudinous. Existing course recommendation systems suggest courses based on either collaborative or content based approach. This work focuses on building an effective Course Recommendation System (CRS) for college students, suggesting the most relevant course based on their learning ability and their preferred choice. In this paper, a rule based approach which addresses the pitfalls and loopholes of the existing technology is suggested. Rule based approach helps to recommend a course better than the existing course recommendation systems.
\end{abstract}

\section{Keywords}

Electives, graduation, classification, personal interest, success rate, students

\section{INTRODUCTION}

Education is an emerging discipline. With the world developing at such a rapid pace, there are number of avenues that are emerging and countless possibilities to learn and upgrade. With everything upgrading at such a tremendous pace, there is also a need for individuals - especially students to equip themselves with the latest technologies. As a result of this, learning options are also growing and a lot of universities are also recognizing this fact and are adding new subjects and courses into the curriculum. But for a fresher or a college student in their teenage years to choose a subject/elective which is not only useful in the future, but also fits into the student's caliber is a difficult choice.

Choosing the right course in the formative years is very important step as the future depends on this decision. Student might not be matured enough to take the right decision and selecting the wrong course means a massive mismatch in the student's aptitude, capability and personal interest. Students have a wavering mind-set and sometimes choose courses which sound sophisticated, but doesn't match with their intelligence levels. This result in the student getting overburdened and as a result of this, they might start lagging behind.

A recommendation system addresses the issue perfectly. Since there is no other solution that is said to provide an accurate analysis of the student's choice and capability, a recommendation system comes handy as it is built so as to recommend the perfect course. There have been countless recommendation systems which pledge to recommend courses to students. But most of them fail to acknowledge the two main factors which decide the success rate of the student. The first factor-to recommend a course based on the student's choice. The second factor to ensure whether the student is efficient enough to do well in the course they have chosen, failing to which, the system will recommend a course not considering students ability.

The recommendation system which addresses both these factors cumulatively and recommends a course accordingly is capable of yielding maximum success in terms of the student's future. The purpose of this paper is to build a system that concentrates on only student's success history and students' present choice, unlike existing approaches collaborative filtering and content based filtering. Content based system recommends courses based on students interests and collaborative based system recommends courses based on the opinions of similar users.

In this paper, a rule based approach is employed. This way, it enhances the optimality of the solution. A rule-based classification can be used to refer to any classification scheme that makes use of IF-THEN rules for class prediction. We make use of a Rule Induction Algorithm to extract relevant rules from the data which can be done directly using sequential covering algorithms. Rule based systems are domain specific expert systems which makes appropriate deductions and choices. Rule Based Classification is cost efficient and accurate in terms of the end result. The speed of system can be optimized as all parts of the code are known to the user. It also provides results with high accuracy and less error rate. Since the output is dependent on the rules, the output responses are stable and won't result in vague results.

Rule based classification follows the same cognitive process as that of a human and hence results are handcrafted. It is suitable for well-known methods since formal logic is applied. The structure of a rule based expert system provides an effective separation of knowledge base from the inference engine. This makes it possible to develop different applications using the same expert shell system.

\section{RELATED WORK}

Itmazi et.al [1] emphasizes the need and the importance of recommendation systems in everyday life and its applications. The wide range of internet activities which use them are ecommerce, webpages and censorship sectors. The authors of this paper mainly focus the use of Recommendation Systems in regard to learning management systems. It elaborates on the various approaches that can be incorporated so as to suit Learning Management System. The various approaches include - Content Based System, Collaborative Filtering System, Rule based Filtering, Hybrid Recommender system etc. The above methods are explained in detail. This paper is studying and searching the ability to use Recommendation Systems in Learning Management Systems as well as designing a new Recommendation System algorithm to recommend list of suitable courses to students while entering their course. The author suggested a hybrid method using content based, collaborative filtering, and rule based filtering 
and Demographic based system.

Chang et.al [2] proposes a two stage Recommendation System for the prediction of student grades along with a filter for professor rating in Course Recommendation for college students. This research uses student information and professor information as datasets and mean average error and confusion matrix analysis as testing parameters. They found out that recommendation Systems provide highly accurate results for students with higher mean grades. With the advent of flexible curriculum systems and a wide variety of courses, word of mouth no longer serves as a standard for providing accurate information to students regarding which courses to be chosen in which they might demonstrate higher aptitude and interest. The paper recognizes that there is a need to ensure that students make the best use of available data to make better decisions regarding course selection. Generally Recommendation Systems use Collaborative Filtering, Associative Rule based classification or Content based systems. But this system seeks, to recommend courses based on factors such as relevance of course to student's department, to increase quantitatively the aptitude of students in the form of grades and quality control mechanism to filter out courses with poor instruction ratings. In this approach, students getting grades will be placed in similar cluster patterns, by using AIS cluster approach for calculating the affinities between different students. This method has been proven as effective. The values of students in clusters are then used as weights in calculating the predicted values.

Reddy et.al [3] has used weighted cluster, top-k-query, reverse-top-k-query and multidimensional index tree to recommend colleges to students. This ensures that students will be in a situation to choose a college based on the voting. This proposed system works well than the proposed traditional methods as it follows k-means and weighted clustering which provides high computational effect on the whole.

Junnutula Meghanath et.al [4] works on the fact that for developing a recommender system, the recommendations shouldn't be passive. It doesn't propagate the chain of thought which thinks that after taking certain inputs, database must be searched. Rather it supports the idea of having the ability to act dynamically through past inputs. The author suggests a recommendation algorithm based on content based system.

Mahony et.al [5] taking into consideration the current enrolment system that makes difficult for students to locate course options that might best fit their individual interests, emphasizes the benefits of deploying recommendation technology to assist users in finding relevant results. More recently, research has been conducted in developing such technology for course recommender systems. The paper proposes a variation on the widely-used item-based collaborative filtering algorithm. The objective of this module recommender system is to recommend elective modules to students based on the core modules that they have selected.

Aher et.al [6] in their course recommendation system in elearning system suggests the best combination of subjects in which the students are interested. In this paper, they propose a framework for recommendation of courses in the E-learning system. In the approach data is collected from students, for example student enrolment for a specific set of course. After getting data, different combination of algorithms is used and they analyze the suitability of combination applied for recommendation. In this paper they outline the architecture and apply the association rule mining at preliminary stage.
Badarenah et.al [7] presents a collaborative recommender system that recommends university elective courses to students by exploiting courses that other similar students had taken. The proposed system employs an association rules mining algorithm as an underlying technique to discover patterns between courses. Experiments were conducted with real datasets to assess the overall performance of the proposed approach. It recommends courses and specifies expected grades for these courses. Accordingly, the student may take a course that is recommended by the system with an acceptable grade. Typically, students have the choice to take courses from a set of elective courses and in most cases, the students take the advices from other students that took such courses. In this recommendation system, it automatically finds similar students and then applies association rule mining algorithm on their courses to create courses association rules.

Kiratijuta Bhumichitr et.al [8] introduces recommendation system by using collaborative system with two techniques namely Pearson Correlation Coefficient and Alternating Least Square. The system first builds student profiles based on their academic records and then finds the similarity between the new students and existing student profiles using similarity techniques stated above. According to the most similar students record with the existing student the course is recommended.

\section{PROPOSED METHODOLOGY}

This paper proposes a course recommendation system. Course recommendation system is an application prepared with the help of experts where the details of background of the students and their aptitudes help finding a course that is beneficial for future. A rule based classification is being used to solve the above scenario. The term rule-based classification can be used to refer to any classification scheme that makes use of IF-THEN rules for class prediction. For this case, a set of students of a department are taken into consideration to check the working capacity of the approach. As per the academic rules and regulations, a student has to select an elective before the commencement of the third year. A student might/might not be in a position to evaluate his calibre and might end up choosing a subject in which he is not proficient yet. In other instances, a student might not be in a position to analyze the difficulty level of the elective chosen and this may end up with unnecessary consequences in the future.

As a solution to this problem, a methodological approach is devised as stated in figure: 1 which takes into consideration the student's performance in previous semesters as well as the student's choice of elective. This ensures a streamlined and a safe, fool proof way to recommend courses.

Before introducing the students in to this CRS, all the courses available for the students throughout the graduation including electives are broadly categorized into four types of course categories namely - Programming, Conceptual, Logical and Theoretical.

Student is prompted to enroll themselves into CRS application after the completion of second year. Student is guided to the system application where in the details such as student roll number, email id is to be entered. He/she is also prompted to choose three choices from a drop down menu of given electives as shown in figure: 2 . Once, the student enters his/ her preferred choice of selection, immediately, the corresponding subject tag is taken into consideration. If that case fails, the next priority subject is checked and the corresponding grade of the student in that category is chosen and so on. 


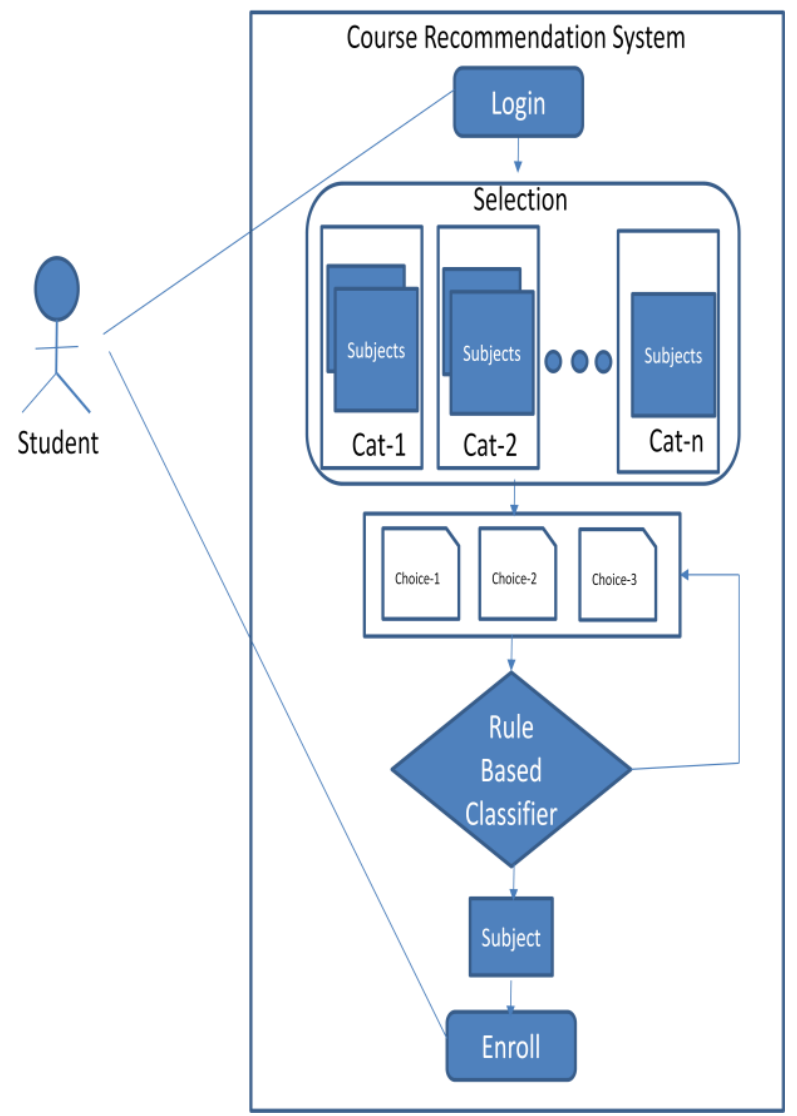

Fig 1: Course Recommendation System

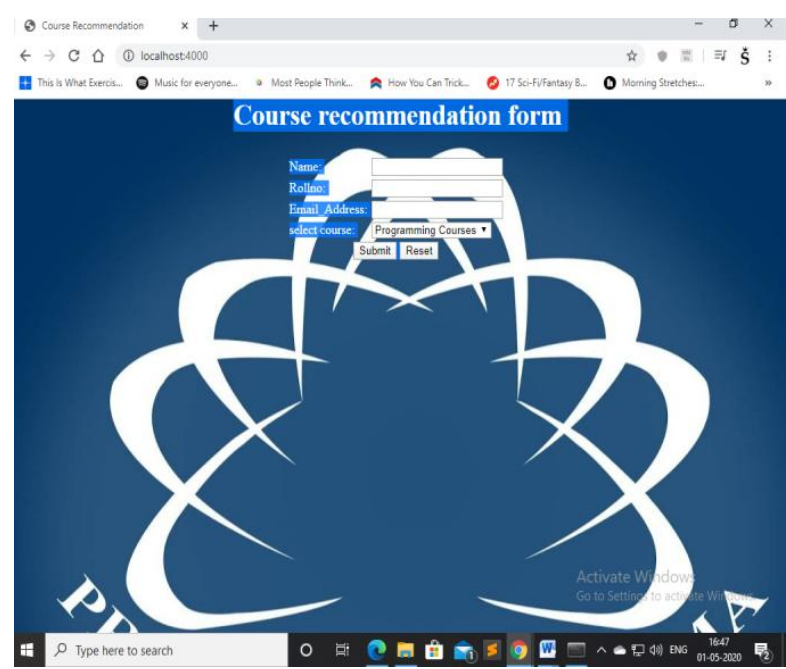

Fig 2: Course Recommendation form for students

Some instances of courses and electives division in to different categories in the experimental data taken are classified into courses such as C, C++, Data Structures, Python and Operating Systems into programming section. Logical Courses include Engineering Mathematics-1, Basics of Electrical Engineering, Engineering Mathematics -2, Engineering Physics, Engineering Drawing, Digital Logic Design, Discrete Mathematical Structures, Probability Statistics and Queuing Theory etc. Conceptual courses include Engineering Chemistry, Data Structures, Computer Organisation, Data communication, Computer Networks, Information Systems Design. Theoretical Subjects include Professional Ethics and Human Values, English, Soft skills lab, Workshop and Environmental Studies. Each of the elective subjects is also categorised into one of the above mentioned categories. Software Testing and Automation (STA), Distributed Operating Systems (DOS), Client Server Technologies (CST) and Technology Management (TM) are the courses available for students to choose from. DOS is a programming course.STA is conceptual. CST is logical and $\mathrm{TM}$ is theoretical.

Supposedly, if a student chooses DOS as elective, then the grades of student's previous semesters consisting of programming subjects are accessed. If, the student has achieved $\mathrm{A}, \mathrm{A}+$ or $\mathrm{O}$ in at-least half or more subjects out of the total subjects, then the student is assigned to that course.

\subsection{STEPS}

1) Login in to the Course Recommendation portal.

2) Select any three choices from the courses available according to priority.

The recommendation system

3) Takes the first choice of student into consideration.

4) With respect to the category of subject, traverse back to the grades of the student in the previous semester following the rules of rule based classifier
a. If the conditions are satisfied according to rule based classifier go to step 5
b. Elseif goto step 4 with the second choice.
c. Else goto Step 4 with third choice.

5) Displays the subject allocated to the student. The role of recommendation system ends

6) Student has to Enroll into the course allocated and logout.

\section{RESULTS}

It has been proven that recommendation system based on Rule based approach is bound to perform better and propose better recommendations than other approaches. The figures 3,4 below show that the students attain better grades through CRS when compared to random allocation methods

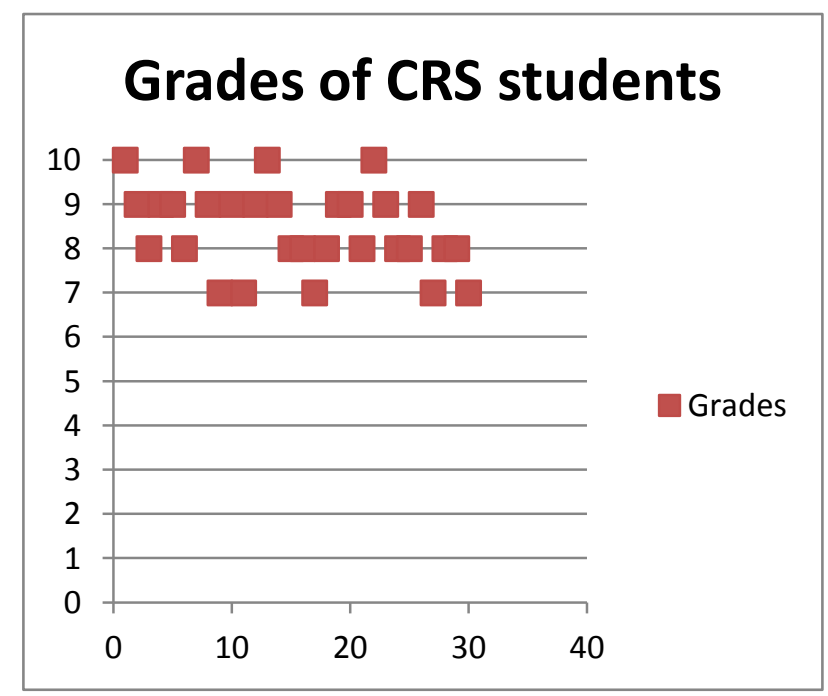

Fig 3: Grades attained by students in subjects allocated through CRS 


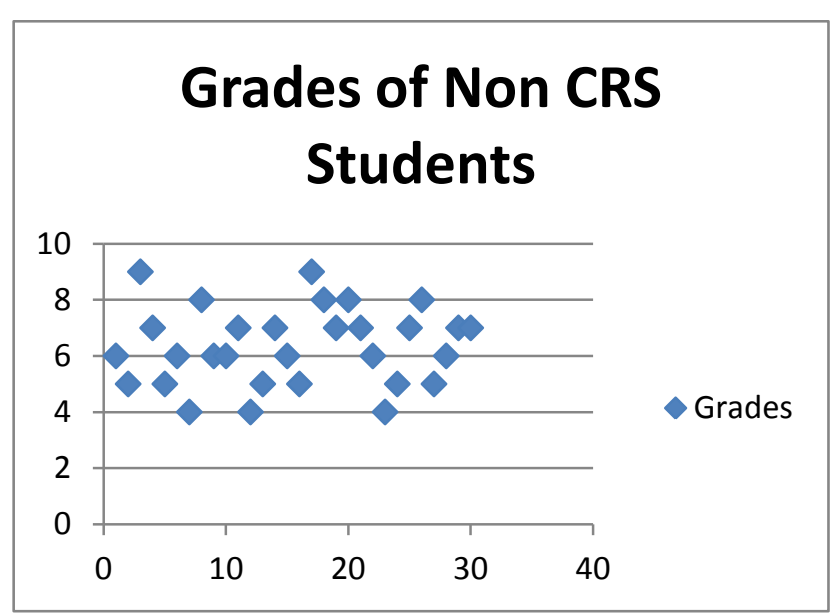

Fig 4: Grades attained by students in subjects allocated through Non CRS

\section{REFERENCES}

[1] A Itmazi, Jamil, and Miguel Megías. "Using recommendation systems in course management systems to recommend learning objects." International Arab Journal of Information Technology (IAJIT) 5.3 (2008).

[2] Chang, Pei-Chann, Cheng-Hui Lin, and Meng-Hui Chen. "A hybrid course recommendation system by integrating collaborative filtering and artificial immune systems." Algorithms 9.3 (2016): 47.
[3] Reddy, Mr Y. Subba, and P. Govindarajulu "College Recommender system using student'preferences/voting: A system development with empirical study." IJCSNS 18.1 (2018): 87.

[4] Reddy, Junnutula Meghanath, and Tengyan Wang. "Online Study and Recommendation System." Final report ACM (2014): 1-8.

[5] O'Mahony, Michael P., and Barry Smyth. "A recommender system for on-line course enrolment: an initial study." Proceedings of the 2007 ACM conference on Recommender systems. 2007.

[6] Aher, Sunita B., and L. M. R. J. Lobo. "A Framework for Recommendation of courses in E-learning System." International Journal of Computer Applications 35.4 (2011): 21-28.

[7] Al-Badarenah, Amer, and Jamal Alsakran. "An automated recommender system for course selection." International Journal of Advanced Computer Science and Applications 7.3 (2016): 166-175.

[8] Bhumichitr, Kiratijuta, et al. "Recommender Systems for university elective course recommendation." 2017 14th International Joint Conference on Computer Science and Software Engineering (JCSSE). IEEE, 2017. 